
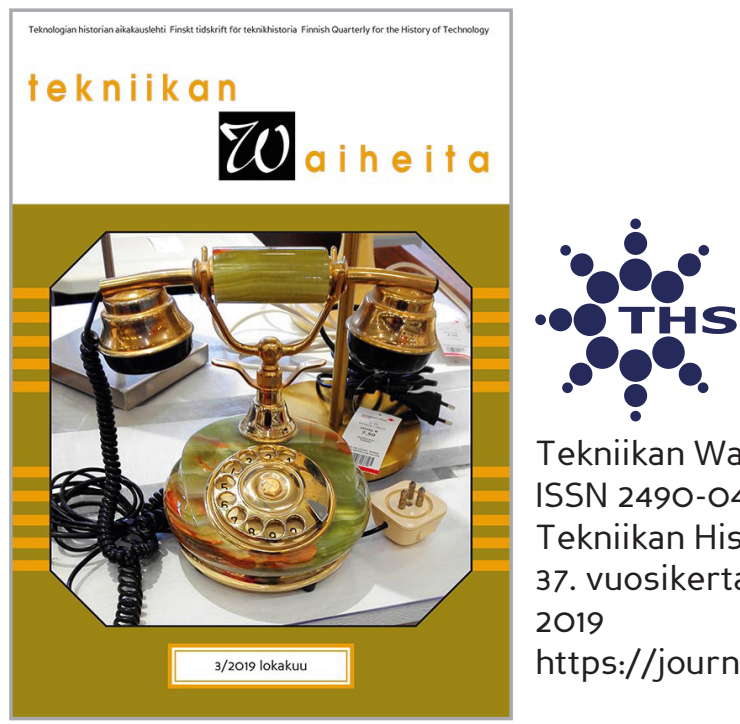

Tekniikan Waiheita

ISSN 2490-0443

Tekniikan Historian Seura ry.

37. vuosikerta:3

2019

https://journal.fi/tekniikanwaiheita

\title{
History and Aesthetics of Progress Indicators
}

Felix Raczkowski \& Mary Shnayien

Mary Shnayien

iD https://orcid.org/0000-0001-5202-1813

To cite this article: Felix Raczkowski \& Mary Shnayien, "History and Aesthetics of Progress Indicators" Tekniikan Waiheita 37, no. 3 /2019): 57-67. https://dx.doi.org/10.33355/ tw. 86775

To link to this article: https://dx.doi.org/10.33355/tw.86775 


\title{
History and Aesthetics of Progress Indicators
}

\author{
Felix Raczkowski' \& Mary Shnayien²
}

The ubiquity of computing brings with it a ubiquity of certain aesthetic forms, of visual conventions and indicators that gradually appear throughout digital cultures. This paper will be concerned with one such convention; one that would appear to be rather misplaced beyond the limited confines of desktop computers and their interfaces: the progress indicator. Progress indicators are continuously changing and modulating their originally limited functions of signifying the progress of human or machinic labor in industrial or computational contexts, to the point where they appear as an aesthetic convention of measurement in popular culture and self-management.

Tracing various forms of progress indicators, this paper will address the shifting status of this element of digital media aesthetics in three steps: First, it will outline a brief media history of progress indicators to clarify the circumstances that enabled this convention in interface design. Second, it will shed light on the various functions fulfilled by progress indicators in today's media environments. Finally, the paper discusses the ramifications of the shift in place, form and function of the progress indicator as evidence of a larger shift in the way machinic and human work are perceived and evaluated in digital cultures.

In 1985, computer scientist Brad Myers presented his paper "The Importance of Percent-Done Progress Indicators for Computer-Human Interfaces" at the Computer Human Interaction conference (CHI). At the time of the conference, one year after the release of the Apple Macintosh, which popularized the graphical user interface for domestic users, the concept of a progress bar was apparently not yet self-explanatory:

Percent-done progress indicators are a technique for graphically showing how much of a long task has been completed. They operate like the giant thermometers in charity drives and "fill up" from empty to full as progress is made. Progress indicators give the user enough information at a quick glance to estimate how much of a task has been completed and when the task will be finished. ${ }^{3}$

Myers can be seen as the first to describe a difference between two types of progress indicators. The percent-done progress indicator he proposes, and the static or rudimentary animated so-called "busy pictures", which were state of the art at the time of his writing and can be considered as the predecessors of what proliferated in the 1990s - especially in web-browsing as seen with the Mosaic and later the Netscape browser - and is known today as a "throbber". Tracing these busy pictures as well as progress bars back to their origin in text-based user interfaces proves difficult due to a lack of documentation.

\footnotetext{
${ }^{1}$ Felix Raczkowski is a post-doc at the department for media studies at the University of Bayreuth. He completed his $\mathrm{PhD}$ on the digitalization of games and play with special focus on gamification and serious games in 2016. His research interests cover game studies, history and aesthetics of digital media, fakes and simulations as well as serialized storytelling.

${ }^{2}$ Mary Shnayien holds a master's degree in media and gender studies and is a PhD candidate at the department for media studies at Ruhr University Bochum. She is a member of the NRW-Forschungskolleg SecHuman - Sicherheit für Menschen im Cyberspace and currently writing her dissertation on the discourse on security and privacy in the post-Snowden era.

${ }^{3}$ Myers 1985, 11.
} 


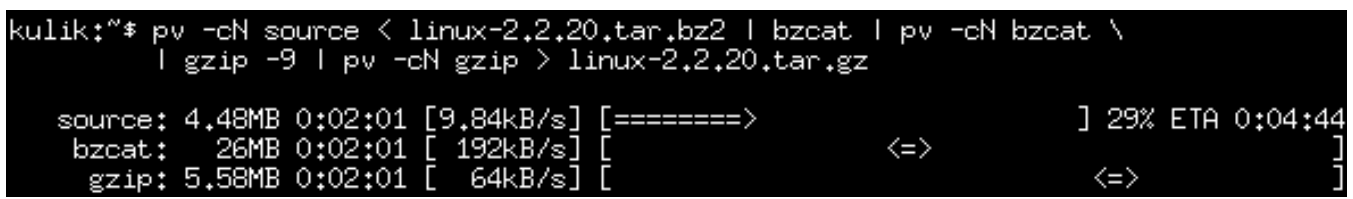

Fig. 1. Demonstration of pv command in a terminal. ${ }^{4}$

What can be observed, however, is that as parts of early UNIX-based systems, some kind of spinning bar was animated in a throbber-like fashion by frequently switching between,$- /, \mid$, and $\backslash$, while progress bars were made out of progressing $=$ signs followed by $\mathrm{a}>$ and a percent-done information to indicate that the machine is busy or working, and to inform users about the status of a given task. When exactly this originated remains unclear, but it can still be seen today, for example on Linux-based operating systems with the "pv" command, which starts a program called "pipe viewer" (Fig. 1).

While the Apple Macintosh's first GUI of 1984 did not feature progress bars, but a throbber in form of the pointer cursor turning into a tiny wristwatch in order to indicate that the machine was working, ${ }^{5}$ later versions of the graphical file manager sported progress bars. In the second half of the 1980s, there even was a project called "SonicFinder", led by William Gaver at Apple Computer, Inc., which was an attempt to augment the standard file manager "Finder" by providing not only a graphical, but also an accompanying auditory user interface. For example, a progress bar visualizing the copying of some files was then accompanied by the sound of pouring water, which would change frequency as the task progressed. ${ }^{6}$

The idea of intentionally adding sound to the graphical interface, which would provide redundant, as well as add new information about the computer's state for the user is based on the notion that sound is an "untapped modality for people" as well as a "seldomexploited resource of computers". ${ }^{7}$ But, most importantly, Gaver notes: "Many people also listen to their computers to get information about mechanisms that cannot be seen" observation that is confirmed in statements like this by Jenifer Tidwell:

I once had a workstation that had a uniquely noisy disk drive, which worked wonderfully (if unexpectedly) as a Progress Indicator for a lot of my software development activities. It wasn't too loud, fortunately, but it did have distinctive sounds for different classes of activities -- one for copying a large file, one for compiling, and so on. If I was waiting for a long compile to finish, I could work on other things without having to watch my monitor; the sudden cessation of the sound told me it was done, in a nice subtle way. ${ }^{?}$

\footnotetext{
4 “Linux pipe viewer." 2017. www.ivarch.com/programs/pv.

5 Jason's Macintosh Museum, "Apple Macintosh 128k (1984) Start Up and Demonstration," YouTube, 01.06.2014, youtu.be/22NC_OURbmo?t=405.

${ }^{6}$ Gaver 1989, 84.

${ }^{7}$ Gaver 1989, 70.

${ }^{8}$ Gaver, 1989, 70.

${ }^{9}$ Tidwell 1999, www.mit.edu.
} 
Today, being mute again, progress bars and throbbers are widely used interface conventions. "Progress Indicators Make a Slow System Less Insufferable", ${ }^{10}$ Katie Sherwin writes on the website of the Nielsen Norman Group, the self-proclaimed "World Leaders in Research-Based User Experience". As user experience and usability research is based mostly on heuristics, or "rules of thumb"11, Sherwin differentiates between various durations of computational tasks. A spinner or throbber should represent everything that occupies the machine for two to ten seconds, and all tasks that take ten seconds or more to complete call for a percent-done animation. This should be done in order to prevent "a great deal of anxiety as the user is uncertain as to whether the computer even received the command in the first place or whether it may have crashed."12

This focus on user experience became apparent in 1995, when the requirements for effective progress indication had coalesced into eight parameters or types of information that Alex Paul Conn recommends for what he calls time affordance ${ }^{13}$. To inform the user of a task's expected duration, the system should offer feedback on the following properties:

1. Acceptance: What the task is and whether it has been accepted with the input parameters or settings

2. Scope: The overall size of the task and the corresponding time the task is expected to take barring difficulties. [...]

3. Initiation: How to initiate the task and, once initiated, clear indication that the task has successfully started

4. Progress: After initiation, clear indication of the overall task being carried out, what additional steps (or substeps) have been completed within the scope of the overall task, and the rate at which the overall task is approaching completion.

5. Heartbeat: Quick visual indication that the task is still "alive" (other indications may be changing too slowly for a quick visual check).

6. Exception: A task that is alive has encountered errors or circumstances that may require outside (i.e. user) intervention.

7. Remainder: Indication of how much of the task remains and/or how much time is left before completion.

8. Completion: Clear indication of termination of the task and the status at termination. How to terminate the task before completion (whether or not errors have occurred). ${ }^{14}$

Conn is mainly concerned with the management of time delays in HCI (Human-Computer Interaction) that are caused by computational tasks and does not mention progress indicators, but the way he proposes to deal with the delays is indebted to Myers' earlier work (which is cited by Conn). Each step in computational operations is supposed to offer clear feedback on the operation's status for the user, although this is not yet framed as userfriendly design, instead emphasizing efficiency in HCI as opposed to later attempts to make the users feel better or to alleviate their anxieties.

\footnotetext{
${ }^{10}$ Sherwin 2014, www.nngroup.com.

${ }^{11}$ Nielsen 2019, www.nngroup.com.

12 Sherwin 2014, www.nngroup.com.

${ }^{13}$ Conn 1995.

${ }^{14}$ Conn 1995,188.
} 
The previous observations indicate that progress indicators in HCI mainly address one issue: mediating the difference between machine time and user time. Apart from preventing anxiety and reassuring the user of a working system, progress indicators, according to Sherwin, "give the user something to look at while waiting", they "offer a reason to wait for the system to finish" and, probably most notably, they "can reduce user's perception of time". ${ }^{15}$ In an ideal world, human computer interaction appears to be seamless; without delay - a paradigm Jack Self criticizes as the "fantasy of 'real-time". ${ }^{16}$ However, as soon as the machine has to complete any more involved computational task or has to handle too many parallel processes, the fundamental difference between the two times becomes apparent. The users have to wait, they cannot act.

Analyzing this fundamental difference, Isabell Otto considers the temporal status of the throbber as one that makes the co-presence of user and machine apparent by bringing about a situation in which they are connected by an element - the discontinuous time - that they both need to overcome. ${ }^{17}$ For Otto, the throbber marks an element in interface-design that enables a media-philosophical reading of human computer interaction as the process of bringing about co-presence between user and machine through the (paradoxical and futile) attempt of seamlessly synchronizing fundamentally different temporal orders. In her assessment of the goals of interface-designers, Otto states that loading bars and throbbers alike are designed and continuously optimized to mediate between the duration of tasks and the waiting users. ${ }^{18}$

What began, as evidenced by Myer and Conn's accounts, with the cybernetic ideal of providing clear feedback about a task's duration or estimated time of completion developed into interface design influenced by cognitive psychology: today's designers do not attempt to convey accurate measurements or estimates, but instead they aim to implement progress indicators that feel fast to make a system appear faster. ${ }^{19}$

This means that it is possible to differentiate between two functions for progress indicators in digital media interfaces. The first one is the cybernetics-inspired accurate measurement or estimation of duration of computational tasks, such as transmissions or calculations. This first function has decidedly analog roots in practices of statistical visualization and scientific management. In the early 20th century, the bar chart is combined with the measurement of time intervals to maximize the use of industrial machinery, ${ }^{20}$ to enhance worker productivity and to track the progress of projects, which is a technique of visualization that is known today after its creator as Gantt charts ${ }^{21}$.

According to Florian Hoof, Gantt charts allow for the kinetic (as opposed to static) modeling of dynamic processes, ${ }^{22}$ which enables consultants like Henry Gantt to track the individual performance of workers ${ }^{23}$. The second and more recent function of progress in-

\footnotetext{
${ }^{15}$ Sherwin 2014, www.nngroup.com.

16 Self 2016.

${ }^{17}$ Cf. Otto 2014.

${ }^{18}$ Cf. Otto 2014.

${ }^{19}$ Cf. Harrison, Zhiquan \& Hudson 2010.

${ }^{20}$ Cf. Gantt 1919, 44-47.

${ }^{21}$ Cf. Hoof 2015, 110-128.

22 Hoof 2015, 167.

${ }^{23}$ Hoof 2015, 111.
} 
dicators appears mostly in networked contexts and is concerned with what we could call the management of boredom or impatience, or, more in line with Otto, the attempt to bridge the difference between two temporalities. This bridging is the goal of efforts in UX research like the ones conducted by Harrison et al. that attempt to design progress indicators in a way that is perceived as less limiting by the users. ${ }^{24}$ The progress bar represents the aesthetic of the first function, while the throbber animates the aesthetic of the second function.

The history of both progress bars and throbbers is evidently connected to digital media interfaces. However, unlike the throbber, the progress bar seems to have become quite popular in a number of different contexts and environments, making it clear that progress bars have become an interface convention, while still maintaining their ambivalence towards what exactly could be expressed by those design elements. In today's media landscapes one can observe many progress indicators that clearly reference the classical computerinterface loading bars, although they are not traditionally associated with digital media interfaces: Since the 2015 remodeling of its corporate design, ${ }^{25}$ the German private TV station "ProSieben" features progress bars to indicate how long a preview or an advertisement is shown until the programming continues. This might not seem odd at first glance, given the overall look and feel of the new interface closely resembles html5-based webpages and was designed to provide a seamless experience between the TV station and its apps on various mobile platforms (cf. Fig. 2).

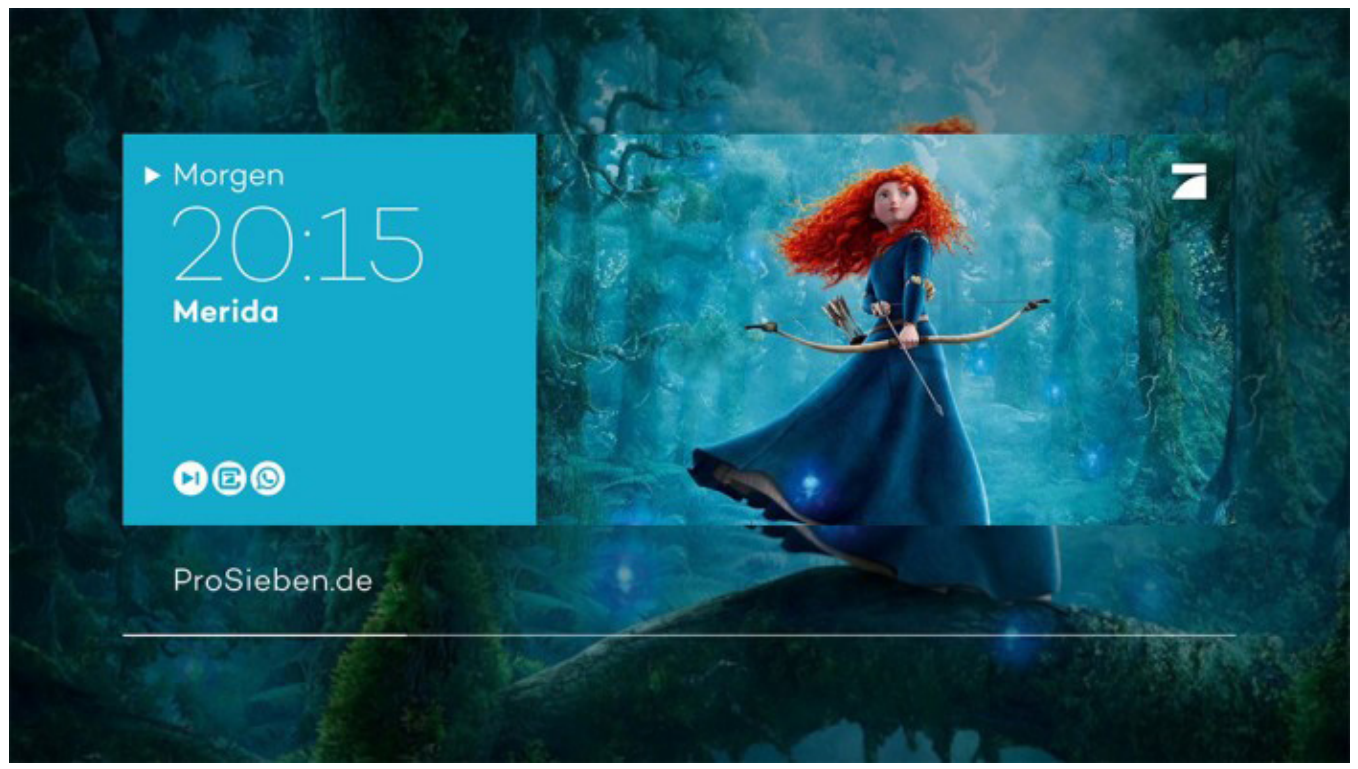

Fig. 2. ProSieben Corporate Design. ${ }^{26}$

\footnotetext{
${ }^{24}$ Cf. Hoof 2015.

${ }^{25}$ Cf. Schaffrinna 2015, www.designtagebuch.de.

26 “ProSieben Corporate Design." 2015. https://www.designtagebuch.de/ab-heute-sendet-prosieben-imneuen-design/prosieben-design-2015-10/.
} 
Nevertheless, those progress bars are employed quite ambiguously: they commonly last for about 8 seconds, which might classify them as throbbers. Thus, they are remarkable: As Winnie Soon notes: "Perhaps, there is a desire in which things would flow continuously, as something like broadcast television." ${ }^{27}$ Drawing on Raymond Williams' concept of the natural break, where the advertisement belongs to the flow of the television program rather than interrupting it, she writes: "[T]elevision exhibits a relatively stable temporality", composed of sequences of program and advertisement, interwoven into an own rhythm, as opposed to networked media, where interruptions cannot be scheduled but are "subjected to $[. .$.$] material conditions at any moment of time." 28$ By introducing progress bars that work as throbbers into the interface, one could argue, the advertisements are framed as an interruption of the flow rather than as a part of it. On the other hand, they help manage boredom and mediate, in Isabell Otto's sense, between two times: the programming and the TV watcher's time.

Progress bars also appear in digital environments without any connection to computational tasks or data transfer, such as in digital self-tracking software, where the steps walked are shown in a progress bar measured against a goal set when using the app for the first time ${ }^{29}$. They function similarly when found in printed documents, such as a calendar in which one can color the months passed by, or in hand-written documents as found largely within the culture of bullet journals. Bullet journals are usually dot-grid paper notebooks in which one tracks one's appointments as well as tasks in a self-designed layout. This renders bullet journals of a mixture of productivity tools following the getting things done-philosophy, Foucauldian self-technology ${ }^{30}$ and attentiveness, often in combination with corresponding Instagram-accounts. Most of them are beautifully crafted, handwritten calligraphy calendars featuring not only To Do's and appointments, but also all kinds of trackers: wellness, spending, workouts, dietary plans, and maybe even watched series or movies, in the form of progress bars.

Although we can read bullet journals as an analogue variety of the self-tracking movement, the bullet journalists enjoy borrowing aesthetic elements derived from early project management visualization, as for example the earlier discussed Gantt charts, as well as their more modern-looking successors in digital aesthetics in the form of progress bars. In bullet journaling, this is not done in order to measure the time of a given task but its progress and to measure the bullet journalist's own habits and work - to the point of rendering things like reading a book or watching a TV series as a task requiring measurable effort.

\footnotetext{
27 Soon 2016, 210.

${ }^{28}$ Soon 2016, 210.

${ }^{29}$ Shnayien 2015, 8 .

${ }^{30}$ Foucault 1993.
} 


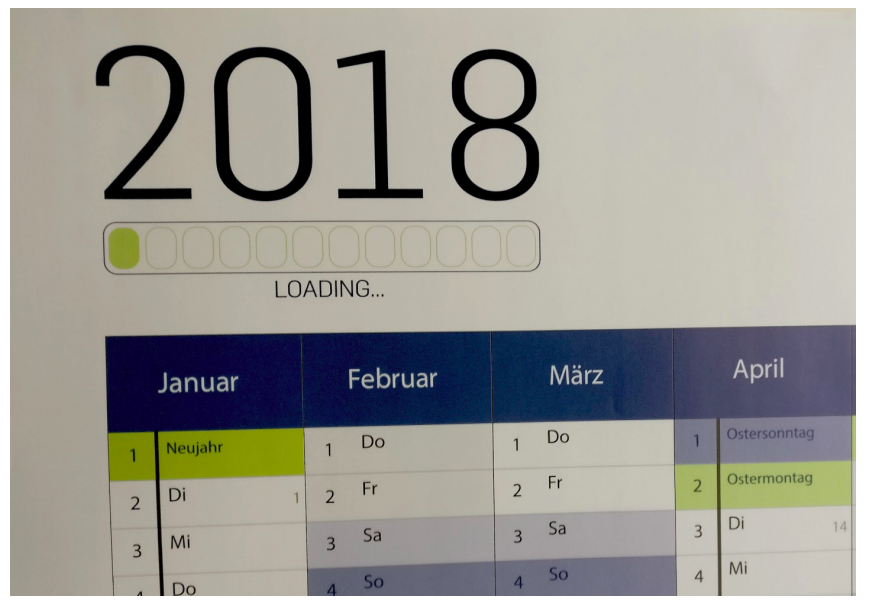

Fig. 3. Calendar with progress indicator for months. Given out by the IT service center of Ruhr University Bochum. Photo by Felix Raczkowski.

It has become apparent that progress bars today - in digital and analogue contexts alike - are very likely to measure the progress of human work instead of just computational tasks. This raises the question concerning the status and the role of the throbber. Contrary to progress bars, throbbers are not appearing outside of digital media interfaces, mainly because they need to be animated to work. One cannot meaningfully draw a throbber or use it to visualize progress in self-tracking applications, as a throbber does not measure progress, but merely denotes it: the throbber imbues digital devices with a certain form of liveliness that goes beyond the mere indication that a device is 'working' (as opposed to crashed). This desire to convey liveliness was originally just one of several concerns for researchers like Conn, who lists the heartbeat as the fifth of eight criteria for the efficient management of time delays. It has since grown to become a major interface element in the form of throbbers, which even carry the heartbeat in their name.

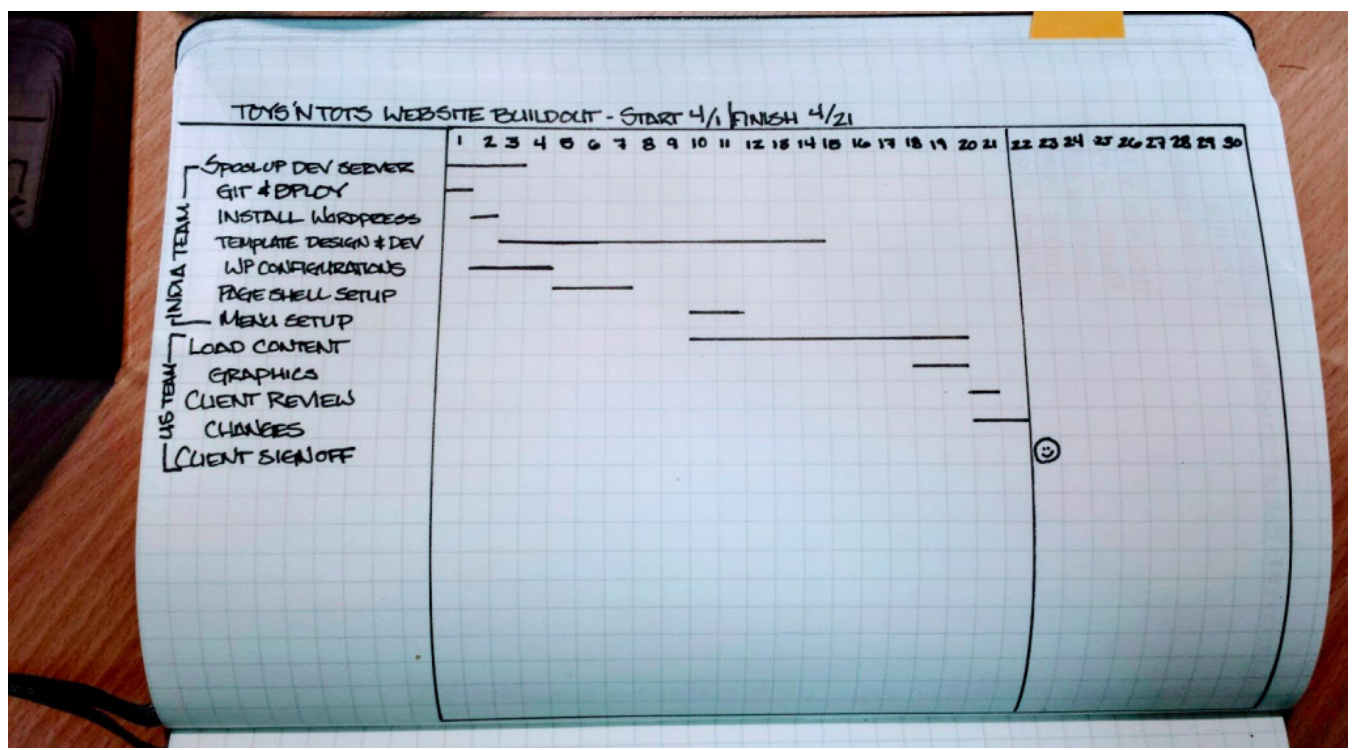

Fig. 4. Gantt chart in a bullet journal. ${ }^{31}$

\footnotetext{
31 “Creating Simple Gantt Charts In Your Bullet Journal”. 2017. Bullet Journals. https://bulletjournals.wordpress.com/2017/04/21/creating-simple-gantt-charts-in-your-bullet-journal
} 
Fig 5. Progress bars in a bullet journal. $^{32}$

According to Uwe Wippich, both the common term of throbber as well as the often pulsating animation associated with it can be read as indicators of life. In his historical work on the mediality of the heart, the pulse - meaning the ebb and flow of beating hearts - is an important indicator for 'life'. Wippich refers to the 16th century physiologist William Harvey as the first person to describe the liveliness of chicken embryos as "an obscure throbbing" 33 . Pulsating and throbbing movement and sound become indicators for life in the life sciences, but they also, and perhaps more importantly, find their way into popular culture as similar indicators for 'living', or at least thinking machines. While HAL 9000's single, menacing camera-eye is still glowing in a continuous red in 1968, by the 1970s and 1980s machinic characters like Battlestar Galactica's Cylons or K.I.T.T. from Knight Rider already signal their liveliness through pulsating, throbbing red lights.

Finally, in contemporary media environments, the Amazon Echo devices and with them Amazon's virtual assistant Alexa draw on all of these aesthetic conventions and associations to present Alexa itself as an active, living household member. Alexa could be called the prime example of a black box in contemporary computing, because the Echo device that forms the end user hardware of Alexa's service offers almost no user interface besides the voice-based interaction. As such, it would be difficult to know whether or not Echo is operational, were it not for the throbber that is built into the top of the device. The blue ring pulsates and glows when Echo is working or talking, operating as the sole visual response of the user interface, thus visualizing Alexa's attentiveness. With assistants like these, an appearance of liveliness becomes especially important, since the Alexa system is supposed to integrate seamlessly into user's everyday life and is continuously marketed by Amazon as a helpful female family member through their TV commercials.

The throbber here is not merely reducing user's anxieties, like Sherwin would probably describe it, nor is it solely a marker of the co-presence of user and machine in a situation of asynchronous temporalities, but it makes Echo, and therefore Alexa, seem vivid. Echo thus becomes a digital device with almost no visual interface that relies not on the precise

\footnotetext{
${ }^{32}$ Instagram.com. 2017. www.instagram.com/p/BRKKpV8hAHL/?taken-by=knitashajanice.

${ }^{33}$ Williams quoted after Wippich 2015, 57.
} 
feedback promised by percent-done progress indicators or similar user experience elements, but instead on an unassuming indicator that fuses computation and liveliness.

To conclude, the paper has demonstrated that there is merit in studying even small interface elements, since it is possible to observe the transformations of digital media and the cultures they inhabit by following these minor changes. Originally conceived as a means to offer feedback to the users of (personal) computers regarding the machine's status during computational operations, progress indicators have become formalized as conventional interface elements during the 1980s.

During this time, research and publications in HCI outline their basic features, which in turn are studied by user interface researchers and designers in the 1990s and 2000s with the goal of making progress indicators more effective. This effectiveness does not necessarily mean a more precise indication of progress, since it also focuses on the manipulation of the user's subjective perception of time and duration. Consequently, the progress bar as an instrument of precise measurement for the duration of computational tasks is becoming less important in digital media interfaces. In line with the standards established by user experience and usability research, the progress bar is often replaced by the throbber, especially in quick, fast-loading media environments.

This development contributes to a shift in the perception of digital devices as well as in their place in relation to their users. The throbber as the second major progress indicator discussed in this paper remains a preeminently digital interface element. It does not measure progress nor does it attempt to educate or inform the user during brief interruptions in real-time interaction. Instead, it could be read as an attempt at something else: the throbber aims to make the machine more relatable, by enhancing it with a certain liveliness. The pulsating animations or lights of throbbers recall the mediation of a pulse and signal that the machine is, in fact, alive and neither dead nor crashed. This also adds another dimension to the problem of the mediation between different temporalities addressed by Otto, since it further connects the user and the machine: both are not only connected by their specific temporal relation, but also because they're positioned as living beings. This liveliness marks especially those machines and devices that are designed to be seamlessly integrated into the everyday life of their users, like for example a digital assistant like Alexa.

Where the progress bar still appears, there is a tendency to make the interruption in the user's experience productive, for example by offering usability tips or explanations of in-depth features during loading times (e.g. the loading bars in many contemporary digital games, or the graphical installer interface of the operating system Ubuntu). However, as has been shown, this does not mean that the progress bar vanishes, but instead, that its place and, more importantly, its function changes: Progress bars appear beyond digital interfaces, while still maintaining their digital aesthetics.

Progress bars are becoming a technique to visualize individual consumption habits or training regimes, they track progress in work and studying and they indicate the duration of advertisements on television. They no longer mainly measure machinic progress, but instead they are also measuring progress in human work - which ties them back to their analogue origins in form of the Gantt chart and other means of scientific management. In this way, it has been shown that the remediation of progress bars that is also transforming their main function into measuring or representing the progress of human work appears to be part of larger shift in the way work is perceived in digital cultures, or, more precisely, most human activity is quantified in a way that resembles work. Thus, the measurement of 
progress can be regarded as an intersection of computer and user (or machine and human), since it not only structures their interaction, but also interfaces the concepts of what human and machinic work might be, thereby constructing conceptual similarities in the perception of humans and machines.

\section{Acknowledgements}

The authors wish to thank Uwe Wippich for his comments on an earlier draft of this paper as well as Moritz Feichtinger, Sarah Kissler and Michael Stevenson for their comments and questions during a presentation of said earlier draft at the conference Digital Cultures: Knowledge / Culture / Technology at Leuphana University Lüneburg in September 2018.

\section{References}

Conn, Alex Paul. 1995. "Time Affordances. The Time Factor in Diagnostic Usability Heuristics." CHI 1995: Mosaic of Creativity: 186-193. https://doi.org/10.1145/223904.223928.

Foucault, Michel. 1993. "Technologien des Selbst". In Technologien des Selbst, edited by Luther H. Martin, 24-62. Frankfurt a.M.: Fischer.

Gantt, Henry L. 1919. Organizing for Work. New York: Harcourt, Brace and Howe.

Gaver, William. 1989. "The SonicFinder: An Interface That Uses Auditory Icons". Human-Computer Interaction 4, no. 1: 67-94. http://dx.doi.org/10.1207/s15327051hcio401_3.

Hoof, Florian. 2015. Engel der Effizienz. Eine Mediengeschichte der Unternehmensberatung. Konstanz: Konstanz University Press.

Harrison, Chris, Zhiquan Yeo, and Scott E. Hudson. 2010. “Faster Progress Bars: Manipulating Perceived Duration with Visual Augmentations". CHI 2010: Pixels and Perception: 1545-1548. https://doi. org/10.1145/1294211.1294231.

Jason's Macintosh Museum - Apple Macintosh 128k (1984) Start Up and Demonstration, https://youtu. be/22NC_OURbmo?t=405.

Myers, Brad. 1985. "The importance of percent-done progress indicators for computer-human interfaces". ACM SIGCHI Bulletin, April 1985: 11-17. http://dx.doi.org/10.1145/317456.317459.

Nielsen, Jakob. 1994. “10 Usability Heuristics for User Interface Design”. Nielsen Norman Group. https:// www.nngroup.com/articles/ten-usability-heuristics/.

Otto, Isabell. 2014. “'Spinning Beach Ball of Death'. Gebräuche der Unterbrechung im Zeitgefüge zwischen Usern und digitalen Medien". lendemains - études comparées sur la France, 39/154-155: 120-134.

Schaffrinna, Achim. 2015. “Ab heute sendet ProSieben im neuen Design." designtagebuch. Last modified 12.02.2015. https://www.designtagebuch.de/ab-heute-sendet-prosieben-im-neuen-design/.

Self, Jack. 2016. "Beyond the self". e-flux journal: Superhumanity. https://www.e-flux.com/architecture/superhumanity/68658/beyond-the-self/.

Sherwin, Katie. 2014. "Progress Indicators Make a Slow System Less Insufferable." Nielsen Norman Group. Last modified 26.10.2014. https://www.nngroup.com/articles/progress-indicators/.

Shnayien, Mary. 2015. "'There's a better version of you out there.' Überwachung, Personalisierung und die Sorge um sich im Selbstversuch“. onlinejournal kultur und geschlecht 2015, no. 15. https://kulturundgeschlecht.blogs.ruhr-uni-bochum.de/wp-content/uploads/2015/08/shnayien_selbstversuch.pdf.

Soon, Winnie. 2016. "Microtemporality: At The Time When Loading-in-progress". Proceedings of the 22nd International Symposium on Electronic Art ISEA2016 Hong Kong: 209-215.

Tidwell, Jenifer. 1999. “Progress Indicator.” Personal Website hosted by MIT. Last modified 17.05.1999. https://www.mit.edu/ jtidwell/language/progress_indicator.html.

Wippich, Uwe. 2015. Schlagen und Wogen. Zur Medialität des ,lebendigen' Herzens. PhD diss. Bochum: RuhrUniversität. 